\title{
MAGNETIC FIELDS IN DISKS AND HALOS OF SPIRAL GALAXIES
}

\author{
RAINER BECK \\ Max-Planck-Institut für Radioastronomie \\ Auf dem Hügel 69 \\ D-5300 Bonn 1 \\ Germany
}

\begin{abstract}
Spiral galaxies host interstellar magnetic fields of 4-15 $\mu \mathrm{G}$ total strength. A significant fraction of the field lines shows large-scale structures. At face-on or moderately inclined view, the field lines run generally parallel to the spiral arms, either with uniform direction with respect to azimuthal angle (axisymmetric spiral, ASS), with one reversal along azimuthal angle (bisymmetric spiral, BSS), or with spiral orientation without dominating direction.

At edge-on view, the field is concentrated in a thin disk, often surrounded by a thick radio disk with field lines mostly parallel to the plane, similar to the quadrupole-type dynamo field. Radio polarization data from NGC891 indicate that the thermal gas seen in $\mathrm{H} \alpha$ is responsible for Faraday depolarization. The required scaleheight of the field of $\sim 4 \mathrm{kpc}$ is comparable to the value expected in case of energy equipartition between magnetic fields and cosmic rays. The interacting edge-on galaxy NGC 4631 shows a much larger radio halo with field lines perpendicular to the disk, possibly driven by a strong galactic wind or the result of a dipole-type halo field.

Field lines bending out of the plane are also visible in face-on galaxies as regions with high rotation measures and low star-formation activity. The resemblance to the phenomenon of the solar corona suggests to call them "galactic coronal holes".
\end{abstract}

\section{INTRODUCTION}

Most phenomena of the active sun emerge from interaction processes between surface and corona magnetic fields (Parker, 1990). Photospheric fields form magnetic loops. These are footpoints of the "streamers" with a slow, dense solar wind. The fast, dilute solar wind originates in coronal holes with unipolar magnetic fields.

It is tempting to compare these phenomena with disk-halo interactions in spiral galaxies. The main difference is that the magnetic energy density dominates out to several solar radii distance while in the disks of spiral galaxies the energy densities of magnetic field, cosmic rays and turbulent gas motion are similar, and the halo field may dominate only above a certain height (see Sect. 4). 


\section{MAGNETIC FIELDS IN DISKS OF SPIRAL GALAXIES}

Linearly polarized radio synchrotron emission offers the most powerful method to study interstellar magnetic fields. While the intensity of the total synchrotron emission yields an estimate of the strength of the total field $B_{t, \perp}$ in the plane of the sky, the intensity of the linearly polarized emission can be used to estimate the strength of the uniform field $B_{u, L}$. The degree of polarization is $275 \%$ in case of a completely uniform field; it decreases with increasing contribution of a random field component. The orientation of the $E$ - vector is intrinsically perpendicular to the orientation of $\mathrm{B}_{\mathrm{u}, \perp}$ but is rotated when the radio wave passes through a magnetized plasma cloud (Faraday rotation). The sign of rotation gives the direction of the field, and the amount of rotation ("rotation measure") is proportional to the average strength of the uniform field $B_{u, \|}$ along the line of sight (see Beck, 1986 and M. Krause, 1990 for details and formulae).

\subsection{Large-scale Field Structures}

Recognition of large-scale field structures requires the analysis of the rotation measures. A uniform field direction yields a periodic variation of rotation measures along azimuthal angle. The phase of such a variation has to be consistent with the pitch angle of $B_{u, \perp}$ and has to change with radius according to the position angle of the spiral arms. Hence this method is not based on a Fourier analysis alone. The assumptions and limitations have been discussed in detail by M. Krause (1990) and Donner and Brandenburg (1990).

For most galaxies the data are still insufficient to determine reliable rotation measures (see Wielebinski, 1990). Only 7 external galaxies have been studied adequately (Table 1). In M31 and IC 342 the dominating field is best described by an axisymmetric spiral (ASS) with constant direction along azimuthal angle and along radius (Beck, 1982; M. Krause et al., 1989a) while M81, M51 (Fig. 1) and possibly M33 show a dominating bisymmetric spiral (BSS) field with one field reversal along azimuthal angle (M. Krause et al., 1989b; Horellou, 1990; Buczilowski and Beck, 1990). In NGC 6946 (Beck et al., 1990, Fig. 2) and probably M83 (Sukumar et al., 1987; Sukumar and Allen, 1989; Sukumar et al., in prep.) the rotation measures are very low in spite of their strong uniform fields: the field in these two galaxies is ordered without a constant direction (neither ASS nor BSS), e.g. forming highly elongated loops or cells.

TABLE 1.

Large-scale structures of disk fields

\begin{tabular}{ccc}
\hline $\begin{array}{c}\text { ASS } \\
\text { (axisymmetric }\end{array}$ & $\begin{array}{c}\text { BSS } \\
\text { spiral) }\end{array}$ & $\begin{array}{c}\text { Neither } \\
\text { (but spiral) }\end{array}$ \\
\hline M31 & M51 & NGC6946 \\
IC342 & M81 & M83? \\
& M33? & Milky Way? \\
\hline
\end{tabular}



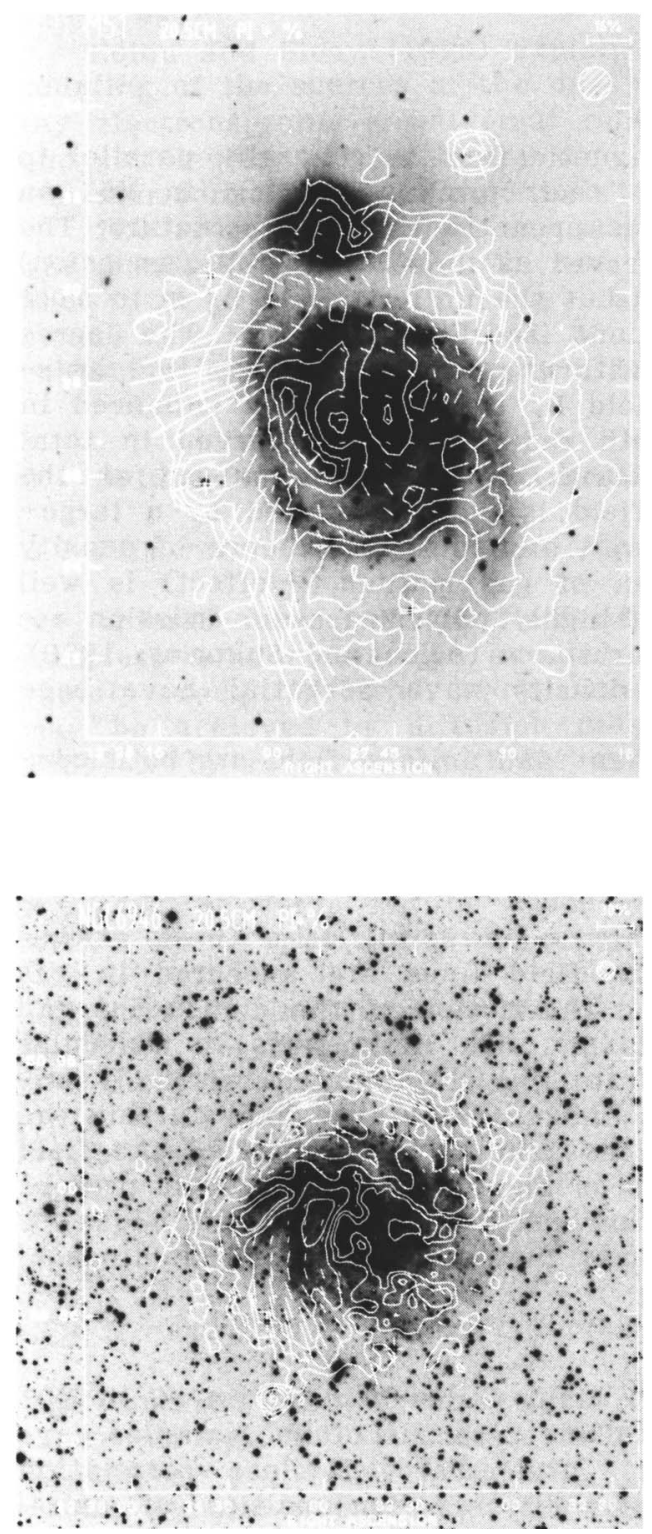

Figure 1. Linearly polarized radio emission from M51 at $\lambda 20.5 \mathrm{~cm}$ observed with the VLA in its D-configuration. The angular resolution is 43 arcsec, the rms noise is $28 \mu \mathrm{Jy} /$ beam. Contours are $120,200,400,800,1600 \mu \mathrm{Jy} /$ beam. The lengths of the observed E-vectors are proportional to the degree of polarization (from Horellou, 1990).

Figure 2. Linearly polarized radio emission from NGC 6946 at $\lambda 20.5 \mathrm{~cm}$ observed with the VLA in its D-configuration. The angular resolution is 42 arcsec, the rms noise is $20 \mu \mathrm{Jy} /$ beam. Contours are 80 , $160,320,640 \mu \mathrm{Jy} /$ beam. The lengths of the observed $\mathrm{E}$ vectors are proportional to the degree of polarization. Faraday rotation at $\lambda 20.5 \mathrm{~cm}$ is almost constant $\sim 37 \mathrm{rad}$ $\mathrm{m}^{-2}$ or $88^{\circ}$, i.e. the vectors approximately show the orientation of the magnetic field component $\mathrm{B}_{\mathrm{u}, \perp}$ (Beck et al., in prep.).

In our own Galaxy there are at least two field reversals between the solar radius and the center (Vallée, 1988). Two inner reversals are consistent with a BSS field structure only if the pitch angle of the field lines is $\leq 6^{\circ}$. However, the pitch angle of the optical spiral arms is $\sim 17^{\circ}$. If this value is also used for the field lines, the field in our Galaxy could resemble the "neither" cases NGC 6946 and M83 without constant 
direction. Alternatively, the field may be axisymmetric with reversals at certain radii (Vallée, 1990).

\subsection{Detailed Field Structure}

The orientation of the interstellar magnetic field is generally parallel to the optical spiral arms. Variations in their pitch angle, e.g. in M51 on the side of its companion galaxy, also appear in the field structure. The unresolved ("turbulent") field $B_{r}$ (observed as unpolarized radio emission) is strongest in the optical spiral arms but the uniform field $B_{u}$ is in most galaxies strongest in the interarm regions (see Figs. 1 and 2). The degree of field uniformity is anticorrelated with the intensity of CO line emission (Beck et al., 1991). The total field $B_{t}$ is only slightly enhanced in the spiral arms, leading to the smooth radio disk as observed in total radio intensity. In well-resolved galaxies, $B_{u}$ is not enhanced at the inner edges of spiral arms, i.e. the field is not compressed by a largescale density-wave shock front. However, an indirect influence of density waves on the dynamo by modification of gas motion ( $\omega$-effect) is well possible (see Sect. 3). In M83 blobs of highly polarized radio emission are observed outside the outer optical spiral arm (Allen and Sukumar, 1990). Systematic motions of clouds in a density-wave potential have been invoked to explain this phenomenon.

M31 is an exceptional case: turbulent and uniform field are both concentrated in the "10 kpc ring" where most of the star formation takes place. A dust lane in the SW quadrant was studied in detail (Beck et al., 1989). A deviation of $\leqslant 25^{\circ}$ from the toroidal field direction occurs near a gas cloud complex.

The general picture of field structure in spiral arms (Beck et al., 1991 ) is based on the idea that the field lines are anchored in gas clouds. The field structure depends on the motion of the clouds but can also influence the cloud motion. Galaxies with little mass in molecular clouds and hence low star formation rate like M31 show a relatively undisturbed field within the spiral arms and no detectable field outside the arms. A large number of clouds produces a high "turbulence" of the field because the superposition of field loops cannot be resolved in present radio maps. Enhanced turbulent motion of the clouds by collisions or turbulence induced by star formation may further tangle the field lines.

\subsection{Field Strengths}

The average strength of the total disk field varies between $\sim 4 \mu \mathrm{G}$ in M31 and M33 and $\sim 12 \mu \mathrm{G}$ in M51 and NGC 6946. A sample of Sbc galaxies gave $\left\langle\mathrm{B}_{\mathrm{t}}\right\rangle \simeq 8 \mu \mathrm{G}$ (Hummel et al., 1988b). The observed close correlation between the radio continuum and far-infrared luminosities of spiral galaxies (e.g. Wunderlich et al., 1987) could mean that the energy density $\mathrm{B}_{t}{ }^{2} / 8 \pi$ of the total field is proportional to the energy density of the stellar radiation field which is dominated by the star formation rate (Hummel et al., 1988b; Völk, 1989). However, the correlation between radio and far-infrared intensities holds within galaxies on scales down to $\sim 1 \mathrm{kpc}$ (Beck and Golla, 1988; Bicay et al., 1989) indicating a cosmic-ray production rate proportional to the star formation rate, plus equipartition 
between cosmic ray and magnetic field energy densities (Chi and Wolfendale, 1990).

Helou and Bicay (1990) explain the radio-FIR correlation by a tight coupling of the sources of the dust-heating photons and those of cosmicray electrons, plus a universal relation between magnetic field strength and gas density of the form $B \propto n^{\beta}(1 / 3 \leq \beta \leq 2 / 3)$. Hence, field strength and star formation rate could also be connected via the gas density.

The star formation process may be controlled by the magnetic field via feedback actions (e.g. Chi and Wolfendale, 1990). Magnetic fields are known to be essential for the stability and fragmentation of molecular clouds (e.g. Bash et al., 1981; Mouschovias, 1990) as well as for cloud collisions (Clifford and Elmegreen, 1983).

\section{ORIGIN OF DISK AND HALO MAGNETIC FIELDS}

\subsection{Primordial Origin ("preserve the order")}

An ordered, protogalactic field is wound up by differential rotation. Complete wind-up can be avoided if the field slips through the clouds rapidly enough (Kulsrud, 1986). Otherwise, a quasistationary configuration may be achieved by diffusion plus dynamo action (Sawa and Fujimoto, 1986 ) or by reconnection (Brett and Kahn, 1990). If the rotation axis is perpendicular to the primordial field, a BSS structure is generated; if the rotation axis is along the primordial field, the result is an ASS structure with a field reversal above and below the plane. An ASS field without reversal, however, can be produced only under special conditions, e.g. with a strong gradient of the primordial field (Sofue, 1990).

\subsection{Local Origin ("preserve the chaos")}

The field is ejected by stars or supernova remnants and wound up by differential rotation (Michel and Yahil, 1973). Field enhancement can be achieved by a local dynamo driven by purely turbulent motion (Molchanov et al., 1985). The resulting field has some alignment, but is subject to frequent reversals within the disk and hence does not show a uniform direction on large scales.

\subsection{Dynamo Models ("order out of chaos")}

ASS and BSS field structures are interpreted as two different azimuthal modes $(m=0$ and 1 ) of the galactic $\alpha \omega$-dynamo (Chiba and Tosa, 1989; F. Krause et al., 1990; Ruzmaikin, 1990; Donner and Brandenburg, 1990). Linear dynamo calculations which assume an axisymmetric gas distribution in galactic disks predict the highest growth rate for the lowest (axisymmetric) mode. A dominating BSS mode cannot be explained by linear dynamo models. Deviations from symmetry or from stationarity may preferably lead to BSS modes:

1. Gravitational disturbances (companion, warping of the disk, density waves, central bars) may excite the BSS mode (Chiba and Tosa, 1990).

2. The dynamo field is growing and still reflects the primordial seed 

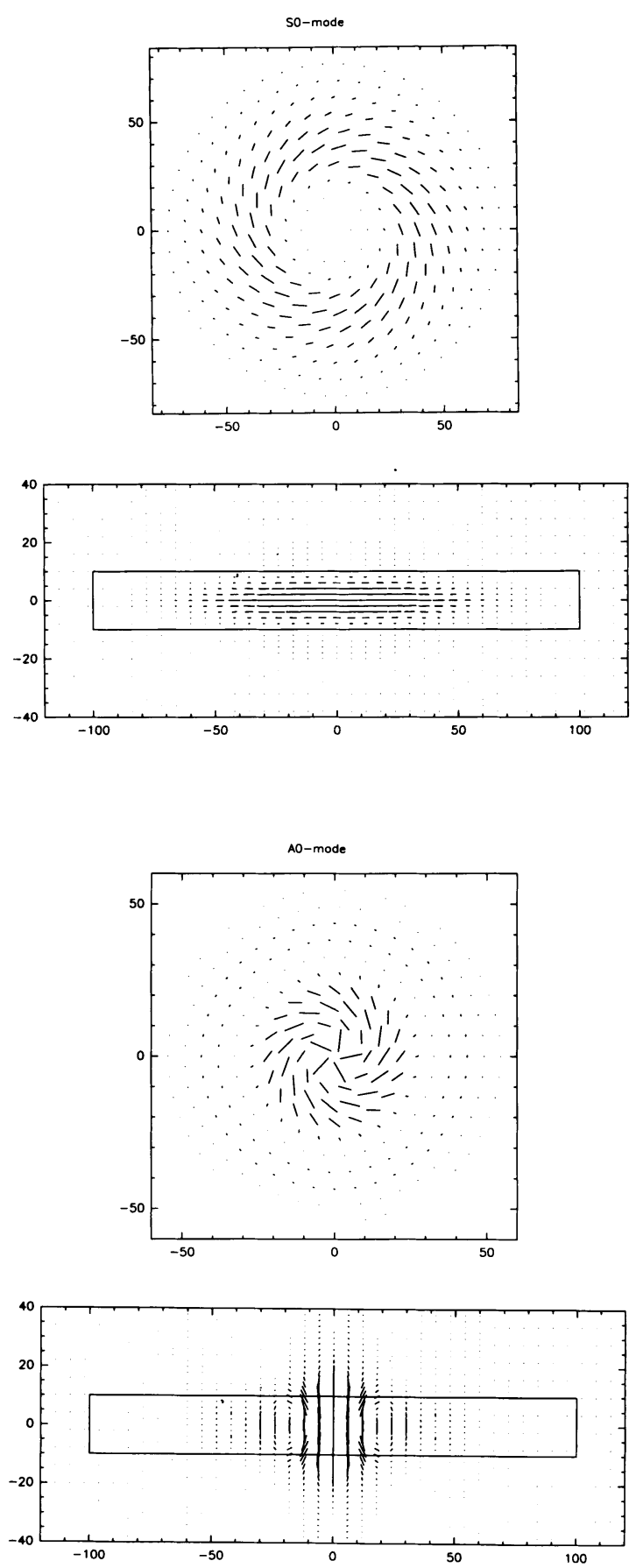

Figure 3. The polarized radio emission from a quadrupole so dynamo field in face-on and edge-on view (Elstner et al., in prep.). The density of cosmic-ray electrons was assumed to be constant. The model galaxy is rigidly rotating until $1 / 3$ of the radius $r$ and differentially rotating at larger radii. The $\alpha$-effect operates only in the disk (up to $r / 10$ height). The conductivity in the halo $(|z|>r / 10)$ is assumed to be $1 / 5$ of that in the disk.

Figure 4. The polarized radio emission from a dipole AO dynamo field in face-on and edge-on view. Assumptions as in Figure 3. 
field which is bisymmetric due to winding-up of the intergalactic or intracluster field (Sawa and Fujimoto, 1986).

3 . The BSS mode appears as the result of strong nonlinear effects for fast-growing dynamo fields (Moss and Tuominen, 1990).

The few available data favor the first possibility. The two BSS cases M81 and M51 have massive companions while the ASS and "zero" cases are accompanied only by small galaxies. The presence of density waves or bars seems to be less important because M83 shows no BSS field.

A superposition of the ASS and BSS mode was proposed for M31 (Sofue and Beck, 1987; Ruzmaikin et al., 1990) but a systematic variation of field strength, plasma density, or pitch angle of the field lines along azimuthal angle may produce a similar effect (M. Krause et al., 1989b).

Dynamo models distinguish between even or odd modes (vertical modes), i.e. no reversal or a reversal of the disk field above and below the plane. The even ASS field (or symmetric $m=0, \mathrm{SO}$ ) can be separated into a toroidal disk field and a quadrupole-type poloidal field in the halo while the poloidal component of the odd ASS field (or asymmetric $m=0$, $A O)$ is of dipole type. Figures 3 and 4 show the polarized radio emission from SO and AO dynamo fields (Elstner et al., in prep.).

In linear theory of the thin-disk $\alpha \omega$-dynamo the ratio of the strengths of the halo and disk field depends on the dynamo numbers $R_{\alpha}$ and $R_{\omega}$ (Ruzmaikin et al., 1988). Flat rotation curves with $\mathrm{v}=\mathrm{v}_{\max }$ yield $B_{h} / B_{d} \simeq\left(\alpha / v_{\max }\right)^{1 / 2}$. For our Galaxy Ruzmaikin et al. (1988) estimated $R_{\omega} \simeq 10, R_{\alpha} \simeq 1$ and $B_{h} / B_{d} \simeq 0.1$. As synchrotron intensity is proportional to $B_{\perp} 1+\alpha_{n t h}\left(\alpha_{n t h} \simeq 1.0\right)$, the halo is normally not expected to contribute much to the radio emission. However, a high star formation rate (high $\alpha$ ) and a low rotation velocity $v_{\max }$ may lead to higher $B_{h} / B_{d}$ and hence to a strong halo field with a large-scale dipole structure (see sect. 4). In the thick-disk dynamo model by Brandenburg et al. (1990) the dipole mode is indeed the easiest to excite.

Linear dynamo theory does not predict the maximum field strength reached in a galaxy. According to Ko and Parker (1989) the dynamo numbers depend on the turbulent velocity which is thought to increase with star formation rate so that the dynamo grows fast - but this does not necessarily mean that the field is strong. The maximum field strength is probably related to the star formation rate (see Sect. 2.3).

Even and odd dynamo fields can be distinguished with help of radio polarization observations:

I. The rotation measure in odd disk fields is one half of those in even disk fields. Comparison between the strengths of the uniform field as derived from polarized radio emission and from rotation measures indicate that the even ASS mode (SO) is more probable in M31 and IC 342. In M51 the rotation measures (corrected for inclination) are too low compared with the polarized synchrotron emission so that an odd BSS mode (A1) may exist (Horellou et al., in prep.).

II. In face-on or moderately inclined galaxies the dipole-type halo field induces high rotation measures in addition to the periodic rotation measures due to the disk field. This effect can be determined by comparison with the rotation measures of nearby extragalactic radio 
sources. However, neither in M51, nor in M81, nor in IC 342 excess rotation measures have been found.

III. The quadrupole-type halo field points perpendicular to the disk only near the center. Near the Galactic center and the centers of active galaxies there is increasing evidence for perpendicular fields (Sofue, 1990) which may originate in central gaseous rings (Lesch et al., 1989). However, the central region of IC 342 shows no z-component between 2 and $5 \mathrm{kpc}$ radius at $250 \mathrm{pc}$ linear resolution (Krause et al., in prep.). The perpendicular field component must be restricted to even smaller radii or is too weak to be observable.

IV. In edge-on galaxies the halo field is directly observable (Sect. 4). The quadrupole-type field is expected to have a small scaleheight and runs mostly parallel to the disk (Fig. 3) while the dipole-type field is more extended and runs perpendicular to the disk (Fig. 4).

\section{MAGNETIC FIELDS IN HALOS OF SPIRAL GALAXIES}

Edge-on galaxies often show vertical dust lanes which may indicate uniform magnetic field lines (Sofue, 1987). Their first detection via polarized radio emission in NGC 4631 by Hummel et al. (1988a) induced a systematic search in several nearby edge-on galaxies. NGC 891 (Hummel et al., $1990 \mathrm{~b}$ ), NGC 3628 (M. Krause et al., in prep.), NGC 4565 (Sukumar and Allen, in prep.) and NGC 5775 (Golla and Beck, 1990; Fig. 5) and several other edge-on galaxies (Hummel, 1990; Hummel, this volume) do not possess extended radio halos but thick disks with typically $\sim 1 \mathrm{kpc}$ scaleheights. In most of these galaxies the observed E-vectors are preferably perpendicular to the disk (Table 2). The same result has been obtained for NGC 4945 (Harnett et al., 1989) and NGC 1808 (Dahlem, 1990) but the polarized emission is restricted to two regions on both sides of the plane. Another galaxy with possibly perpendicular E-vectors is NGC 253 (Klein et al., 1983). However, the inclination is too low to exclude a dominant contribution from the disk.

In view of the high frequencies and the regular structure of the polarized emission Faraday rotation is probably small so that most of the field lines run parallel to the disk. In the disk itself the polarized emission is negligible due to Faraday depolarization.

NGC 891 is the only edge-on galaxy with polarization data at two

TABLE 2 .

Large-scale structures of thick disk or halo fields

\begin{tabular}{cccc}
\hline $\begin{array}{c}\text { B I disk } \\
\text { (quadrupole?) }\end{array}$ & $\begin{array}{c}\text { B } \perp \text { disk } \\
\text { (dipole?) }\end{array}$ & $\begin{array}{c}\text { B I I disk } \\
\text { (outflow?) }\end{array}$ & $\begin{array}{c}\text { No thick disk } \\
\text { detected }\end{array}$ \\
\hline NGC 253? & NGC 4631 & NGC 1808 & M31 \\
NGC 891 & & NGC 4945 & \\
NGC 3628 & & & \\
NGC 4565 & & & \\
NGC 5775 & & & \\
\hline
\end{tabular}



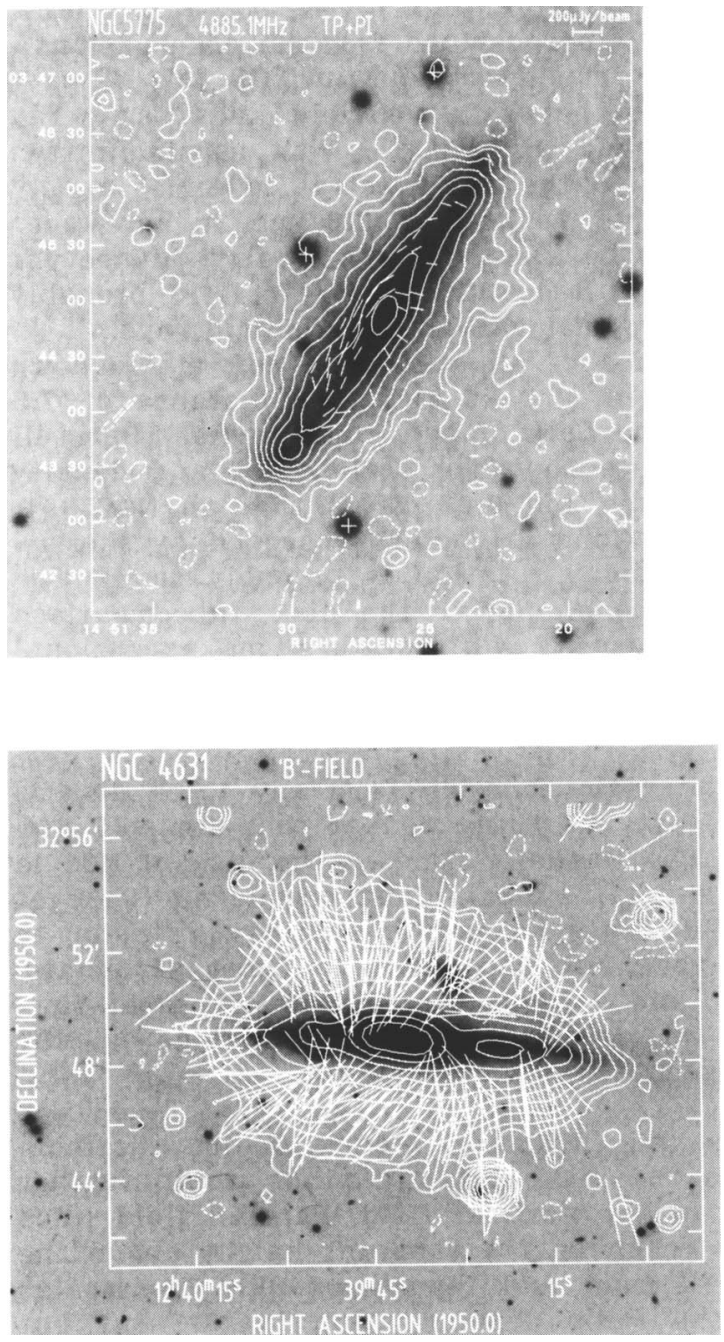

Figure 5. Total radio emission from NGC 5775 , observed at $\lambda 6.1 \mathrm{~cm}$ with the VLA in its D-configuration. The synthesized HPBW is 12.5 , the rms noise $25 \mu \mathrm{Jy} /$ beam. Contour levels are $40,80,160, \ldots$ $\mu \mathrm{Jy} / \mathrm{beam}$. The vectors are E-vectors turned by $90^{\circ}$ and approximately show the orientations of the magnetic field lines because foreground Faraday rotation is low. The vector lengths are proportional to the polarized intensity (from Golla and Beck, 1990).

Figure 6. Total radio emission from NGC 4631 at $\lambda 20.2$ $\mathrm{cm}$, observed with the VLA in its $\mathrm{D}$-configuration. The angular resolution is 40 arcsec, the rms noise is $40 \mu \mathrm{Jy} /$ beam. Contours are 100,200, $400, \ldots \mu \mathrm{Jy} /$ beam. The vectors are E-vectors turned by $90^{\circ}$ and approximately show the orientations of the magnetic field lines because foreground Faraday rotation is low (from Hummel et al., 1990a).

frequencies (Hummel et al., 1990a; Allen and Sukumar, 1990). The field lines are again parallel to the plane but flaring out at the edges of the disk (Allen and Sukumar, this volume). A similar behaviour is visible in some cases of a quadrupole dynamo field (see Fig. 3).

The increase of the degree of polarization with height above the disk of NGC 891 has been analyzed by Hummel et al. (1990a). The data can be well modelled by Faraday depolarization in a thermal gas of $\sim 1 \mathrm{kpc}$ scaleheight (assuming a distance of $7.2 \mathrm{Mpc}$ ) and a turbulent magnetic field of $\sim 4 \mathrm{kpc}$ scaleheight. The scaleheight of the thermal gas as derived from the radio data agrees well with that observed in $\mathrm{H} \alpha$ light (Rand et al., 
1990; Dettmar, 1990). The fitted thermal electron density in the plane of $\left\langle\mathrm{N}_{\mathrm{e}}\right\rangle \simeq 0.03 \mathrm{~cm}^{-3}$ indicates a filling factor of $\mathrm{f}=\left\langle\mathrm{N}_{\mathrm{e}}\right\rangle / \mathrm{N}_{\mathrm{cloud}} \simeq 0.06$. Both $\left\langle\mathrm{N}_{\mathrm{e}}\right\rangle$ and $\mathrm{f}$ are lower than the values derived from $\mathrm{H} \alpha$ observations.

The scaleheight of the turbulent magnetic field in NGC 891 of $z_{B} \simeq$ $4 \mathrm{kpc}$ is consistent with equipartition between the field and cosmic ray energy densities where $z_{B}=2 z_{C R}=\left(3+\alpha_{n t h}\right) z_{s y n}$. With a synchrotron scaleheight of $\mathrm{z}_{\mathrm{syn}} \simeq 0.9 \mathrm{kpc}$ and a nonthermal spectral index of $\alpha_{\mathrm{nth}} \simeq$ 1.0 (Hummel et al., 1990b) equipartition means $\mathrm{z}_{\mathrm{B}} \simeq 3.6 \mathrm{kpc}$, in agreement with the polarization data. This is the first observational evidence for equipartition in the halo of a galaxy. Hence the magnetic field probably extends much farther out than the thermal gas and the cosmic rays.

NGC 4631 (Fig. 6) is the only case of an extended radio halo known until now. The synchrotron scaleheight of $\sim 1.9 \mathrm{kpc}$ at a distance of 7.5 $\mathrm{Mpc}$ is about twice as large as for the bulk of edge-on galaxies (Hummel, 1990). The depolarization data indicate that the scaleheight and density of thermal gas is also higher $\left(-1.3 \mathrm{kpc}\right.$ and $\left.0.07 \mathrm{~cm}^{-3}\right)$ than in NGC 891 . The distribution of polarized emission and E-vectors observed by Hummel et al. (1988a) is so regular that Faraday rotation is probably small. The magnetic field lines (Fig. 6) are perpendicular to the disk. In comparison to all other edge-on galaxies observed, NGC 4631 is an exceptional galaxy. A survey of 181 edge-on galaxies observed with the Effelsberg and VLA radio telescopes (Hummel et al., 1990c) disclosed no other case with a halo similar to NGC 4631 .

The other extreme is M31: The radio emission from any thick disk is not detectable and has to be at least 200 times weaker than for NGC 891 (Berkhuijsen et al., this volume). The magnetic field in the disk of M31 is almost toroidal with only weak deviations near cloud complexes (Beck et al., 1989). The lack of vertical field lines hampers cosmic ray diffusion to high $\mathrm{z}$ and may account for the weak emission there. In terms of dynamo models (Sect. 3.3) a low $\alpha$-effect and a high rotation velocity lead to a low ratio $B_{\text {halo }} / B_{d i s k}$. However, this is not true for all regions in $M 31$ (see Sect. 5).

Models of Parker field loops (e.g. Urbanik, 1990) predict the halo field lines to be perpendicular to the disk. Galactic winds would further increase the degree of uniformity of the vertical field. Parallel field lines can be obtained if all field loops reach only a constant height above the disk, e.g. by galactic chimneys (Norman and Ikeuchi, 1989). The scaleheight is sufficient to explain the radio depolarization in NGC 891 but not in NGC 4631 (see above).

The predominance of magnetic fields parallel to the disk (Table 2) can be best interpreted in terms of dynamo theory. Quadrupole-type dynamo fields can explain both the extension and the field structure at high z (Fig. 3). The dipole-type field of NGC 4631 may be the result of high star formation and low rotation velocity (see Sect. 3.3 and Fig. 4).

The high star formation in NGC 4631 may also drive a galactic wind which pushes field lines out of the galactic plane. Extensions of the radio halo in regions of uniform field (Fig. 6) correspond to the "streamers" of the solar corona. If the halo field is in energy equipartition with the cosmic rays, it has a scale height of as large as $\sim 8 \mathrm{kpc}$. Beyond $8 \mathrm{kpc}$ height above the plane of NGC 4631 , where Faraday 
depolarization becomes negligible, the degree of polarization decreases again. A similar effect is seen in NGC 891. This indicates an increase of field turbulence with increasing height, as predicted by some galactic wind models (Breitschwerdt et al., 1990).

Convection or diffusion of field lines from the disk into the halo has not yet been treated by dynamo models. On the other hand, galactic wind models still have to take the magnetic field into account. As the scaleheight of $\mathrm{B}^{2}$ (at least in NGC 891) is higher than that of the thermal gas, the magnetic pressure will dominate above a certain height.

\section{GALACTIC CORONAL HOLES}

A region in the SE quadrant of M31 with vertical field lines (Berkhuijsen et al., this volume) coincides with low HI emission and with a hole in the $\mathrm{CO}$ ring at $10 \mathrm{kpc}$ radius (Dame et al., 1991). Regions with little gas content may allow the field to leave the galactic disk and form open field lines - as in the case of solar coronal holes.

In face-on galaxies coronal holes are observable as regions of high rotation measures with neither enhanced plasma density ( $\mathrm{H} \alpha$ emission) nor enhanced field strength (total synchrotron emission). The maps of IC 342 (Krause et al., 1989a) and also of M51 (Horellou, 1990) seem to show such phenomena. The hole in $\mathrm{M} 51$ is visible as an extended minimum in polarized emission between the spiral arms traced by $\mathrm{H} \alpha$ and $\mathrm{CO}$ (Fig. 1). This region has to be studied with higher resolution.

Of special interest is the SW quadrant of NGC 6946 (Fig. 2): Strong Faraday depolarization and high Faraday rotation measures without indications for enhanced plasma density or uniform field strength allow to conclude that a large fraction of the disk field bends out into the halo. The rotation measures are almost constant over NGC 6946 ("neither" case in Table 1), except in the SW quadrant where both high and low values occur (Beck et al., in prep.). The spiral arms in the SW quadrant of NGC 6946 are more diffuse and the $\mathrm{H} \alpha$ emission is weaker (Bonnarel et al., 1986) compared with the rest of the galaxy: The galactic coronal hole occurs in a region of low star-forming activity.

\section{FUTURE OBSERVATIONS}

Radio polarization data of both face-on and edge-on galaxies hold the clue to understand the magnetic disk-halo connections. Rotation measures will help to distinguish between the various models (Table 3) while highresolution polarization observations will show the role of gas complexes and star-forming regions.

Observations in $\mathrm{CO}$ lines are required to investigate the relation between cloud distribution and turbulence and the field structure in and above the disk. Optical and X-ray detection of warm and hot halo gas, together with radio polarization data, would allow to determine the halo field strength and its energy density and to compare it with the energy densities of gas and cosmic rays. Finally, the detection of optical polarization caused by the Davis-Greenstein mechanism would directly 
TABLE 3 .

Observation of magnetic disk-halo connections

\begin{tabular}{|c|c|c|c|c|}
\hline & $\begin{array}{l}\text { Edge-on ga } \\
\text { degree of } \\
\text { polarization }\end{array}$ & $\begin{array}{l}\text { laxies } \\
\text { rotation } \\
\text { measure }\end{array}$ & $\begin{array}{c}\text { Face-on ga } \\
\text { degree of } \\
\text { polarization }\end{array}$ & $\begin{array}{l}\text { laxies } \\
\text { rotation } \\
\text { measure }\end{array}$ \\
\hline $\begin{array}{l}\text { Dynamo fields } \\
\text { Galactic winds } \\
\text { "Coronal holes" }\end{array}$ & $\begin{array}{l}\text { high } \\
\text { medium } \\
\text { high }\end{array}$ & $\begin{array}{l}\text { medium } \\
\text { low } \\
\text { low }\end{array}$ & $\begin{array}{l}\text { low } \\
\text { low } \\
\text { low }\end{array}$ & $\begin{array}{l}\text { medium } \\
\text { low } \\
\text { high }\end{array}$ \\
\hline
\end{tabular}

indicate the field orientation with very high resolution. New CCD polarimeters are promising (Scarrott et al., 1990; Neininger et al., 1990).

\section{CONCLUSIONS}

a. The analysis of radio polarization data shows that interstellar magnetic fields have both a large-scale component with uniform direction as well as small-scale components related to gas clouds and star-formation processes.

b. The disk field has an axisymmetric, a bisymmetric or a "neither" structure, in most cases without a reversal above/below the plane.

c. The thick disk field has a parallel, perpendicular or "neither" orientation with respect to the disk, with a clear dominance of parallel fields.

d. Field lines perpendicular to the disk seem to produce a huge radio halo - but this case is very rare.

e. Disk and halo fields can best be interpreted in terms of dynamo models of mostly quadrupole type. The influence of galactic winds or fountains on the (small-scale ?) field structure is not yet understood. Future measurements of Faraday rotation are required.

f. We could learn a lot from solar magnetic fields (Table 4).

TABLE 4.

Magnetic Phenomena

\begin{tabular}{|c|c|}
\hline Sun: & Galaxies: \\
\hline $\begin{array}{l}\alpha \omega \text {-dynamo } \\
\text { flux tubes } \\
\text { loop prominences } \\
\text { reconnection } \\
\text { solar wind } \\
\text { streamers (slow wind) } \\
\text { ? } \\
\text { coronal transients } \\
\text { coronal holes (fast wind) }\end{array}$ & $\begin{array}{l}\alpha \omega \text {-dynamo, } \alpha^{2} \text {-dynamo } \\
\text { Verschuur's helices? (this volume) } \\
\text { Parker instabilities } \\
\text { ? } \\
\text { galactic wind (NGC } 4631 \text { ) } \\
\text { extensions into the radio halo (NGC4631) } \\
\text { chimneys } \\
\text { outflow? (NGC } 1808, \text { NGC 4945) } \\
\text { galactic coronal holes }\end{array}$ \\
\hline
\end{tabular}




\section{REFERENCES}

Allen, R.J., Sukumar, S. (1990) Ap. J. (in press)

Bash, F., Hausman, M., Papaloizou, J. (1981) Ap. J. 245, 92

Beck, R. (1982) Astr. Ap. 106, 121

Beck, R. (1986) IEEE Trans. on Plasma Science PS-14, 740

Beck, R., Golla, G. (1988) Astr. Ap. 191, L9

Beck, R., Loiseau, N., Hummel, E., Berkhuijsen, E.M., Gräve, R., Wielebinski, R. (1989) Astr. Ap. 222, 58

Beck, R., Buczilowski, U.R., Harnett, J.I. (1990) in Galactic and Intergalactic Magnetic Fields, eds. R. Beck, P.P. Kronberg, R. Wielebinski, Kluwer, Dordrecht, p. 213

Beck, R., Berkhuijsen, E.M., Bajaja, E. (1991) in Dynamics of Galaxies and Molecular Cloud Distribution, IAU Symp. No. 146, eds. F. Combes, F. Casoli, Kluwer, Dordrecht

Bicay, M.D., Helou, G., Condon, J.J. (1989) Ap. J. 338, L53

Borınarel, F., Boulesteix, J., Marcelin, M. (1986) Astr. Ap. Suppl. 66, 149

Brandenburg, A., Tuominen, I., Krause, F. (1990) Geophys. Astrophys. Fluid Dynamics 50, 95

Breitschwerdt, R., McKenzie, J.F., Völk, H.J. (1990) Astr. Ap. (in press)

Brett, L., Kahn, F. (1990) in The Interstellar Disk-Halo Connection in Galaxies, Poster Proc. IAU Symp. No. 144, ed. H. Bloemen, Leiden Observatory, p. 81

Buczilowski, U.R., Beck, R. (1990) Astr. Ap. (in press)

Chi, X., Wolfendale, A.W. (1990) M.N.R.A.S. 245, 101

Chiba, M., Tosa, M. (1989) M.N.R.A.S. 238, 621

Chiba, M., Tosa, M. (1990) in Galactic and Intergalactic Magnetic Fields, eds. R. Beck, P.P. Kronberg, R. Wielebinski, Kluwer, Dordrecht, p. 131

Clifford, P., Elmegreen, B.G. (1983) M.N.R.A.S. 202, 629

Dahlem, M. (1990) Ph.D. thesis, University of Bonn

Dame, T., Thaddeus, P., Koper, E. (1991), in Dynamics of Galaxies and Molecular Cloud Distribution, IAU Symp. No. 146, eds. F. Combes, F. Casoli, Kluwer, Dordrecht

Dettmar, R.-J. (1990) Astr. Ap. 232, L15

Donner, K.J., Brandenburg, A. (1990) Astr. Ap. (in press)

Golla, G., Beck, R. (1990) in The Interstellar Disk-Halo Connection in Galaxies, Poster Proc. IAU Symp. No. 144, ed. H. Bloemen, Leiden Observatory, p. 47

Harnett, J.I., Haynes, R.F., Klein, U., Wielebinski, R. (1989) Astr. Ap. 216 , 39

Helou, G., Bicay, M.D. (1990), in Galactic and Intergalactic Magnetic Fields, eds. R. Beck, P.P. Kronberg, R. Wielebinski, Kluwer, Dordrecht, p. 239

Horellou, C. (1990) Diplomarbeit, University of Bonn

Hummel, E. (1990) in Windows on Galaxies, eds. G. Fabbiano et al., Kluwer, Dordrecht, p. 141

Hummel, E., Lesch, H., Wielebinski, R., Schlickeiser, R. (1988a) Astr. Ap. 197, L29

Hummel, E., Davies, R.D., Wolstencroft, R.D., van der Hulst, J.M., Pedlar, A. (1988b) Astr. Ap. 199, 91

Hummel, E., Beck, R., Dahlem, M. (1990a) Astr. Ap. (in press) 
Hummel, E., Dahlem, M., van der Hulst, J.M., Sukumar, S. (1990b) Astr. Ap. (in press)

Hummel, E., Beck, R., Dettmar, R.-J. (1990c) Astr. Ap. (in press)

Klein, U., Urbanik, M., Beck, R., Wielebinski, R. (1983) Astr. Ap. 127, 177

Ko, C.M., Parker, E.N. (1989) Ap. J. 341, 828

Krause, F., Meinel, R., Elstner, D., Rüdiger, G. (1990) in Galactic and Intergalactic Magnetic Fields, eds. R. Beck, P.P. Kronberg, R. Wielebinski, Kluwer, Dordrecht, p. 97

Krause, M. (1990) in Galactic and Intergalactic Magnetic Fields, eds. R. Beck, P.P. Kronberg, R. Wielebinski, Kluwer, Dordrecht, p. 187

Krause, M., Hummel, E., Beck, R. (1989a) Astr. Ap. 217, 4

Krause, M., Beck, R., Hummel, E. (1989b) Astr. Ap. 217, 17

Kulsrud, R. (1986) in Plasma Astrophysics, ESA SP-251, p. 531

Lesch, H., Crusius, A., Schlickeiser, R., Wielebinski, R. (1989) Astr. Ap. 217, 99

Michel, F.C., Yahil, A. (1973) Ap. J. 179, 771

Molchanov, S., Ruzmaikin, A., Sokolov, D. (1985) Sov. Phys. Usp. 28, 307

Moss, D., Tuominen, I. (1990) Geophys. Astrophys. Fluid Dynamics 50, 113

Mouschovias, T.Ch. (1990) in Galactic and Intergalactic Magnetic Fields, eds. R. Beck, P.P. Kronberg, R. Wielebinski, Kluwer, Dordrecht, p. 269

Neininger, N., Beck, R., Backes, F. (1990) in Galactic and Intergalactic Magnetic Fields, eds. R. Beck, P.P. Kronberg, R. Wielebinski, Kluwer, Dordrecht, p. 253

Norman, C.A., Ikeuchi, S. (1989) Ap. J. 345, 372

Parker, E.N. (1990) in Galactic and Intergalactic Magnetic Fields, eds. R. Beck, P.P. Kronberg, R. Wielebinski, Kluwer, Dordrecht, p. 1

Rand, R.J., Kulkarni, S.R., Hester, J.J. (1990) Ap. J. 352, L1

Ruzmaikin, A. (1990) in Galactic and Intergalactic Magnetic Fields, eds.

R. Beck, P.P. Kronberg, R. Wielebinski, Kluwer, Dordrecht, p. 83

Ruzmaikin, A., Sokoloff, D., Shukurov, A. (1988) Nature 336, 341

Ruzmaikin, A., Sokoloff, D., Shukurov, A., Beck, R. (1990) Astr. Ap. 230, 284

Sawa, T., Fujimoto, M. (1986) P.A.S. Japan 38, 133

Scarrott, S.M., Rolph, C.D., Semple, D.P. (1990) in Galactic and Intergalactic Magnetic Fields, eds. R. Beck, P.P. Kronberg, R. Wielebinski, Kluwer, Dordrecht, p. 245

Sofue, Y. (1987) P.A.S. Japan 39, 547

Sofue, Y. (1990) in Galactic and Intergalactic Magnetic Fields, eds. R. Beck, P.P. Kronberg, R. Wielebinski, Kluwer, Dordrecht, p. 227

Sofue, Y., Beck, R. (1987) P.A.S. Japan 39, 541

Sukumar, S., Allen, R.J. (1989) Nature 340, 537

Sukumar, S., Klein, U., Gräve, R. (1987) Astr. Ap. 184, 71

Urbanik, M. (1990) in The Interstellar Disk-Halo Connection in Galaxies, Poster Proc. IAU Symp. No. 144, ed. H. Bloemen, Leiden Observatory, p. 111

Vallée, J.P. (1988) A. J. 95, 750

Vallée, J.P. (1990) Ap. J. (in press)

Völk, H.J. (1989) Astr. Ap. 218, 67

Wielebinski, R. (1990) in The Interstellar Medium in Galaxies, eds. H.A. Thronson, J.M. Shull, Kluwer, Dordrecht, p. 349

Wunderlich, E., Klein, U., Wielebinski, R. (1987) Astr. Ap. Suppl. 69, 487 